\title{
SOIL MOISTURE RETRIEVAL WITH S-BAND SAR DATA
}

\author{
Raffaella Guida ${ }^{a}$, Vasillis Fotias ${ }^{b}$

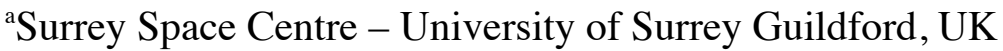 \\ r.guida@surrey.ac.uk \\ ${ }^{\mathrm{b}}$ Aratos Technologies S.A, Greece \\ vfotias@aratos.gr
}

\begin{abstract}
In June 2014 an airborne Synthetic Aperture Radar (SAR) flight campaign was run in the south UK in order to acquire S-band datasets on several scenarios and for different applications in preparation for the launch of the first UK SAR mission NovaSAR-S. On request, X-band data could be acquired concurrently.

This paper shows some of the results of the research project AS14-12 aimed at assessing S-band performance in retrieving soil moisture in bare or poorly vegetated areas. Comparison with $\mathrm{X}$-band dataset was also among the objectives and, at this purpose, the Integral Equation Model (IEM) Inversion through an Artificial Neural Network (ANN) was considered a suitable technique at both frequencies and applied. Retrieval results at both frequencies are here presented against ground truth collected on the site concurrently with the airborne datasets. They show that, for the application considered, the retrieval from S-band data is more accurate than that from X-band with an average error smaller than $4 \%$.
\end{abstract}

Index Terms- Soil moisture, Synthetic Aperture Radar, S-band, Integral Equation Model, Artificial Neural Network.

\section{INTRODUCTION}

In the past decade Synthetic Aperture Radar (SAR) was successfully applied to soil moisture retrieval with promising results and the recent launch of dedicated space missions carrying a radar sensor is evidence of its increasing importance. An example is represented by the Soil Moisture Active Passive (SMAP) mission, designed by the US National Aeronautics and Space Administration (NASA) and launched in January 2015. SMAP carries both a radiometer and a SAR in L-band to improve both resolution and accuracy in the soil moisture retrieval. Another mission having soil moisture characterization among its potential application fields is the UK NovaSAR-S. Apart from the Chinese Huanjing-1C, there is no other availability of S- band SAR data today and this explains the need of demonstration campaigns to test the performance of S-band SAR data on a number of targeted applications, soil moisture retrieval included, before the NovaSAR-S satellite will be launched.

This paper contains some of the results of a larger project on the analysis and development of SAR applications for NovaSAR-S and its main focus is to investigate the capability of the S-band SAR to retrieve soil moisture in bare or poorly vegetated soils. For this purpose SAR data were acquired from the Airbus Airborne SAR Demonstrator, in both S- and X-band, under research directions later specified. Coordination with Airbus guaranteed that, concurrently, ground truth on the selected site was acquired as part of the project's objectives and consisted of soil moisture and roughness parameters. As expected the ground truth collection was a really time-consuming task while, especially on extended areas, the availability of satellite data-images might bring to the development of moisture maps on large scale. However the reliability of these maps has been only partially investigated and this paper aims to contribute to this discussion too.

\section{METHODOLOGY}

The methods commonly used to date to retrieve soil moisture from SAR images are model-based. These methods represent a general approach to retrieve soil moisture using microwaves measurements, as they are not site-dependent and can be easily adapted to different experimental conditions handling single or multi-specification SAR data. Despite of many research contributions in the last years, soil moisture retrieval from SAR images stays a laborious procedure with the main issue being how to isolate the information of interest due to the strong dependence of the backscattering coefficient on the soil properties [1].

The choice of the most appropriate approach depends on a few parameters such as the frequency. In this project the authors aimed at testing SAR performance in S-band but also comparing the results with a concurrent acquisition in $\mathrm{X}$-band at different polarizations. For this purpose, the 


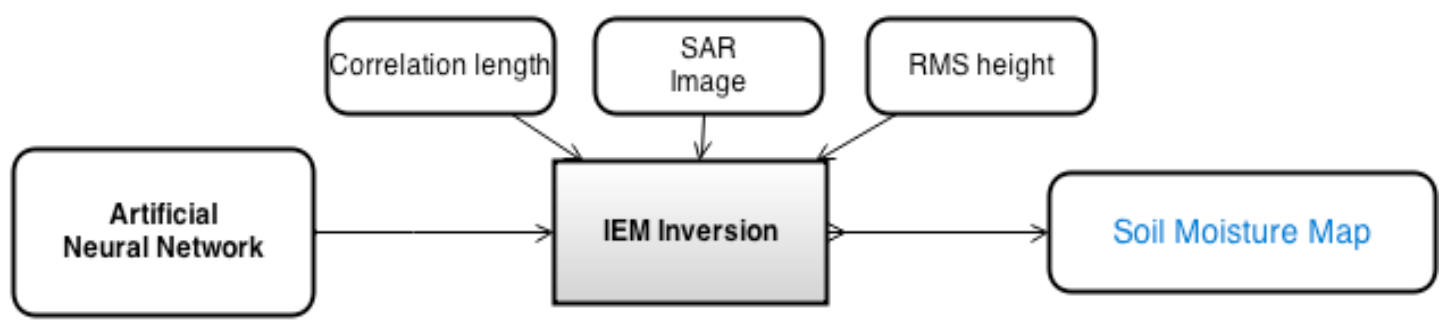

Figure 1 - Schematic diagram of IEM Inversion, ANN and Soil moisture map relation.

Integral Equation Model (IEM) Inversion [2] through an Artificial Neural Network (ANN) was found to be a suitable technique at both frequencies and the rationale follows. The main drawback of the approach is the need to have some prior information about the roughness which had to be included in the operation of ground truth collection.

The IEM, [2], is a direct scattering model which links the backscattering coefficient $\sigma^{\circ}$ to radar parameters, such as the incidence angle and the carrier, and physical ones as the parameters characterizing the surface roughness, i.e. the standard deviation $\sigma$ and the correlation length $l$ of the stochastic process describing the surface height, and the dielectric constant $\varepsilon_{S}$ which summarizes the dielectric features of the soil. It is worth to note that $\varepsilon_{S}$ is a paramater strongly dependent on soil texture and, as a result, volumetric soil moisture affects the dielectric constant. The Hallikainen model [3], gives a polynomial relation between the dielectric constant and the volumetric soil moisture, which coefficients are empirically calculated through a regression analysis by using data between $1.4 \mathrm{GHz}$ and $18 \mathrm{GHz}$.

The ANN used for the IEM inversion is a multi-layer perceptron (MLP). It is a feed-forward network, characterized by a unique unidirectional data flow, without loop; in particular, the MLP is made up of several layers [4]. In this experiment the ANN is trained and used in a later stage as an input for the IEM Inversion as well as the soil roughness parameters and the SAR image as shown in Figure 1. It is worth to note that the IEM Inversion allows the utilization only of $\mathrm{HH}$ and $\mathrm{VV}$ polarizations, so of all SAR channels acquired only these have been used. The training of the network is of massive importance and, supposing that all radar parameters are known, the training phase links the triple $\left(\sigma, l, \sigma^{o}\right)$ to the volumetric soil moisture $m_{v}$. Finally the training quadruple set $\left(\sigma, l, \sigma^{o}, m_{v}\right)$ is used as input for the neural network where the first three parameters of each set are used to calculate the fourth one, $m_{v}$. At the beginning the weights are chosen pseudo-randomly. Finally the trained neural network becomes ready for the IEM Inversion.

\subsection{Instrument}

The Airbus UK Airborne SAR Demonstrator has been used in this project, jointly funded by the Satellite Application Catapult, Airbus, NERC and the Surrey Space Centre. The airborne SAR instrument, in conjunction with a groundbased image processor, is capable of acquiring SAR images from altitudes of between 1500 and $3000 \mathrm{~m}$ in either daylight or darkness, and regardless of whether the scene being imaged is cloud covered. The system currently has XBand $(9.65 \mathrm{GHz})$ and S-Band $(3.2 \mathrm{GHz})$ capabilities with a choice of quad, triple, double or single polarization. It is capable of a wide range of swath width/resolution, with a spatial resolution (finest) of $\sim 18 \mathrm{~cm}$ for the $\mathrm{X}$-Band and $\sim 1$ $\mathrm{m}$ (far) to $\sim 2.2 \mathrm{~m}$ (near) for the $\mathrm{S}-\mathrm{Band}$ [5].

\subsection{Site and Ground Truth acquisition}

The Royal Horticultural Society (RHS) garden at Wisley in the English county of Surrey, south of London, is the area where the airborne data and in situ measurements have been taken. RHS has provided the authors with information about the area and training on how to use Delta-T probes (PR2/4, SM200, SM300 and HH2) for in situ measurements of soil moisture which have been taken concurrently with the SAR data acquisition.

The approach usually used to assess the soil moisture by means of Delta-T devices consists of taking five measurements per every clod of $2 \times 2 m$ following the geometry shown in Figure 2(a), where the red circles represent the points in which the probes must be inserted. Once the five measurements are taken, they are then averaged in order to obtain a unique value, which represents the soil moisture content of the resolution cell of $2 \times 2 m$. For this experiment, due to the lack of time during the acquisition, as the in situ measurements had to be taken concurrently with the SAR data and the soil moisture is a fast varying parameter, the approach was slightly modified for sake of simplicity and a lower number of measurements were taken according to the grid shown in Figure 2(b).

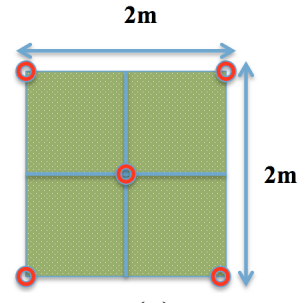

(a)

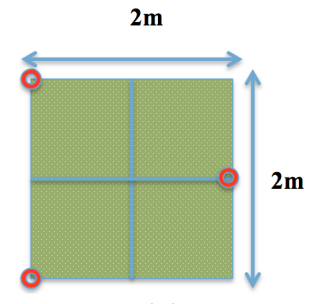

(b)
Figure 2 - Geometry of sampling for the ground truth measurement on clods of $2 \times 2 \mathrm{~m}$. 
In order to apply the inversion of the IEM-ANN, also the RMS height and the correlation length of the surface need to be known. There are different instruments to measure the soil roughness with the most accurate being the laser Profilometer. In this study a more common and less expensive mechanical Profilometer has been used, made of steel needles $1 \mathrm{~cm}$ far apart and inserted in a metallic frame positioned vertically against the ground, Figure 3 . The height of the needles in the structure is tuned and fixed to reflect the soil surface profile beneath and, when this has been done for each needle, the profilometer is laid down on a piece of paper (Fig.3) where a curve of points representing the soil profile can be drawn to later derive RMS and correlation length.

\subsection{SAR data}

Given the proximity with London and consequent restrictions to the height of flight for security reasons, not all operating modes of the Airbus demonstrator could be offered on this area. Restrictions to the height of flight (about $730 \mathrm{~m}$ ) limited the swath width to $640 \mathrm{~m}$ and, as a consequence, was not possible to capture the entire area of interest in one shot. Since different sites were selected for this exercise, the acquisition of two SAR strips were designed and the flight plan was organized so that all areas were imaged from the same direction. The acquisition took place on the 24th June 2014, at two frequencies (S- and Xbands) and four polarization modes ( $\mathrm{HH}, \mathrm{VV}, \mathrm{HV}, \mathrm{VH})$.

The specific areas where the ground truth was collected were selected according to the following criteria: presence of bare or poorly vegetated soil; flat terrain in order to avoid effects from topography; quite isolated area, without the presence of large buildings or trees; accessible and permit areas for the probes-instruments installation.

\section{RESULTS}

In Figure 4 a portion of the S-Band SAR amplitude image in $\mathrm{VV}$ polarization is shown (Fig.4a), together with the corresponding soil moisture map (Fig.4b) retrieved by applying the methodology previously explained. The results of the same procedure applied to the X-band VV-polarized dataset is also shown for comparison in Fig.4c.
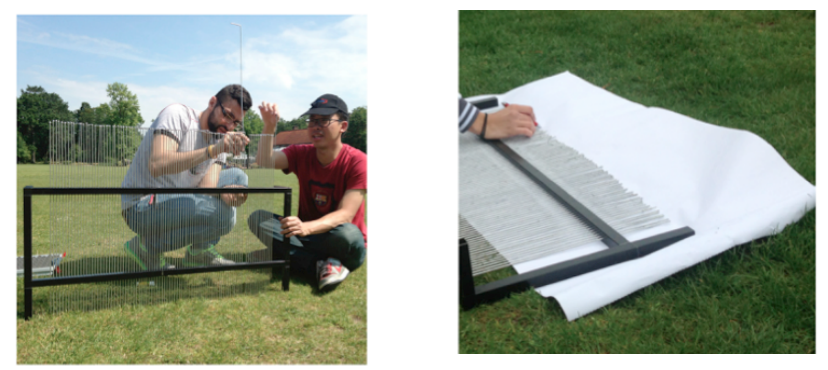

Figure 3 - Profilometer adopted to measure the soil roughness on the site of study.
It is notable that in both maps there is a large number of black pixels, representing two possible situations: the first one includes pixels with soil moisture values out of the range of interest, between $50 \%$ and $100 \%$, which is unlikely to occur under the weather conditions observed on the day; the second includes meaningless pixels (those in the ranges ]$-\infty 1.9 \%]$ or $[101 \%+\infty[)$. Physically these values cannot be assumed as soil moisture values and as a result they are discarded from the experiments estimations. In order to make meaningful comparison with the ground truth collected, only some areas in the image with moisture values in the range $2-50 \%$ were selected. Seven of these sites were selected for the S-band image and four for the X-band. Their soil moisture and the overall averages are indicated in Tables I and II, respectively for the S- and the X-band. The results show, at average, a better agreement between the moisture values locally measured and those retrieved from the S-band SAR dataset with an average error smaller than $4 \%$ which, according to the RHS scientist involved in the project, is considered generally acceptable for areas where the soil moisture ranges between $25 \%$ and $35 \%$. The corresponding retrieval with the X-band dataset shows an error in the order of $10 \%$. Results in $\mathrm{HH}$ polarization show the same trend.

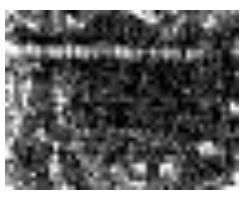

(a)

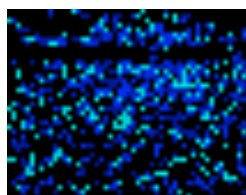

(b)

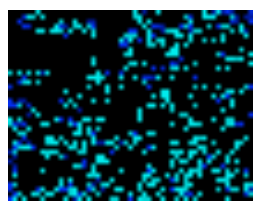

(c)

\section{$[2-10] \%$ \\ $[10-20] \%$ \\ $[20-30] \%$ $[30-40] \%$ \\ $[40-50] \%$}

Figure 4 - (a) SAR amplitude image in S band; (b) Soil moisture map in S-band VV polarization; (c) Soil moisture map in $\mathrm{X}$-band $\mathrm{VV}$ polarization.

TABLE I - S-Band

\begin{tabular}{|c|c|c|c|}
\hline Samples & Area $m^{2}$ & $\begin{array}{c}\text { Estimated } \\
\text { soil moisture } \\
\widetilde{\boldsymbol{m}}_{v}\end{array}$ & $\begin{array}{c}\text { Ground truth } \\
m_{v}\end{array}$ \\
\hline 1 & 32 & $33.78 \%$ & $25.95 \%$ \\
\hline 2 & 24 & $31.92 \%$ & $31.38 \%$ \\
\hline 3 & 48 & $26.60 \%$ & $26.82 \%$ \\
\hline 4 & 32 & $29.06 \%$ & $30.39 \%$ \\
\hline 5 & 36 & $27.02 \%$ & $22.06 \%$ \\
\hline 6 & 48 & $30.97 \%$ & $21.08 \%$ \\
\hline 7 & 56 & $28.21 \%$ & $22.38 \%$ \\
\hline & $\begin{array}{c}\text { Total } \\
\text { Area } m^{2}\end{array}$ & Average $\widetilde{\boldsymbol{m}}_{v}$ & Average $\boldsymbol{m}_{v}$ \\
\hline & 276 & $29.65 \%$ & $25.88 \%$ \\
\hline & Error & \multicolumn{2}{|c|}{ Standard deviation $\sigma$} \\
\hline & $-3.77 \%$ & 12.62 & 4.1 \\
\hline
\end{tabular}


TABLE II - X-Band

\begin{tabular}{|c|c|c|c|}
\hline Samples & Area $\boldsymbol{m}^{\mathbf{2}}$ & $\begin{array}{c}\text { Estimated } \\
\text { soil moisture } \\
\tilde{\boldsymbol{m}}_{\boldsymbol{v}}\end{array}$ & $\begin{array}{c}\text { Ground truth } \\
\boldsymbol{m}_{\boldsymbol{v}}\end{array}$ \\
\hline $\mathbf{1}$ & 16 & $26.98 \%$ & $34.36 \%$ \\
\hline $\mathbf{2}$ & 16 & $21.22 \%$ & $32.20 \%$ \\
\hline $\mathbf{3}$ & 16 & $8.91 \%$ & $24.92 \%$ \\
\hline $\mathbf{4}$ & 16 & $21.95 \%$ & $23.78 \%$ \\
\hline \multirow{3}{*}{$/$} & $\begin{array}{c}\text { Total } \\
\text { Area } \boldsymbol{m}^{2}\end{array}$ & Average $\widetilde{\boldsymbol{m}}_{\boldsymbol{v}}$ & Average $\boldsymbol{m}_{\boldsymbol{v}}$ \\
\cline { 2 - 4 } & 64 & $19.01 \%$ & $28.81 \%$ \\
\cline { 2 - 4 } & Error & \multicolumn{2}{|c}{ Standard deviation $\boldsymbol{\sigma}$} \\
\cline { 2 - 5 } & $9.80 \%$ & 12.56 & 5.39 \\
\hline
\end{tabular}

\section{CONCLUSIONS}

The study described and discussed in this paper aimed to investigate the capability of the S-band SAR to retrieve soil moisture in bare or poorly vegetated fields. For this purpose SAR data were acquired from the Airbus Airborne SAR Demonstrator, in both S- and X-band, concurrently with the ground truth on selected sites, in order to make a comparison between performance at different frequencies for this kind of application. IEM Inversion model has been applied through ANN and results show a very good performance in S-band, better than in X-, with an average error smaller than $4 \%$ which is considered generally acceptable for areas where the soil moisture ranges between $25 \%$ and $35 \%$. Hence, consideration of soil moisture retrieval as application for the future NovaSAR-S is advised.

As future work, the authors aim to apply the same study to dry areas-fields in order to investigate S-band performance also for very low range of soil moisture values.

\section{ACKNOWLEDGEMENTS}

The authors thank Dr Tijana Blanusa, RHS Fellow, for her invaluable support and advice throughout the experiment, and the Satellite Applications Catapult, Airbus Defense and Space, NERC for providing the datasets used in this study.

\section{REFERENCES}

[1] F.T.Ulaby, "SAR Biophysical Retrievals: Lessons Learned And Challenges To Overcome", Proceedings of the 2nd International Workshop on Retrieval of Bio\& Geo-Physical Parameters from SAR Data for Land
Applications, ESA-ESTEC, (Nordwijk, The Netherlands), 21-23 October 1998.

[2] A.K.Fung, Z.Li, K.S.Chen,"Backscattering from a randomly rough dielectric surface," IEEE Trans. Geosci. Remote Sens., vol. 30, no. 2, pp. 356-369, Mar. 1992.

[3] M. T. Hallikainen, F. T. Ulaby, M. C. Dobson and M. A. El-Rayes, "Microwave Dielectric Behavior of Wet Soil - Part I: Empirical Models and Experimental Observations," IEEE Transactions on Geoscience and Remote Sensing, vol. GE-23, no. 1, pp. 25-34, January 1985.

[4] G. Satalino, F. Mattia, M. W. J. Davidson, T. Le Toan, G. Pasquariello and M. Borgeaud, "On Current Limits of Soil Moisture Retrieval from ERS-SAR Data," IEEE Transactions on Geoscience and Remote Sensing, vol. 40, no. 11, pp. 2438-2447, November 2002.

[5] Catapult, Astrium, NERC (2013) AirSAR User Information Meeting, Available at: https://sa.catapult.org.uk/documents/7820487/0/2111 AirSAR_UserInformationMeeting_211113_Final_std.p df/f404134e-ddee-4269-967e-187ff8b6ee1b (Accessed: 12 March 2014). 